\title{
A numerical study of crack shielding and deflection under
} extensive plasticity

\author{
Allan Burke-Veliz, Philippa A. S. Reed and Stavros Syngellakis* \\ School of Engineering Sciences, University of Southampton, Southampton, SO17 1BJ, U.K.
}

\begin{abstract}
Experimentally observed crack deflection events in multi-layered material systems, occurring even under pure mode-I loading, are here simulated and explained through elasto-plastic finite element modelling. The crack tip opening displacement is adopted as the crack driving force and estimated along crack paths whose deflection is predicted using the maximum tangential strain criterion. Shielding conditions that promote deflection and bifurcation are evaluated. It is shown that, under conditions of extended plasticity, CTOD evolution as a crack approaches an interface can reveal crack shielding and amplification, and that crack deflection and growth can be assessed from the variation of tangential strains around the crack tip.
\end{abstract}

Keywords: Multi-layered systems; crack deflection; crack path bifurcation; crack growth; plasticity; finite element analysis

\footnotetext{
${ }^{*}$ Corresponding author. Tel.: +44 23 80592844; fax: +44 2380594813

E-mail address: ss@soton.ac.uk (S. Syngellakis)
} 


\section{Introduction}

Fatigue failure has been studied extensively to prevent components of engineering systems from going prematurely out of service or causing damage to the whole system. More demanding service conditions have been met with the introduction of tougher materials and complex architectures but they have also resulted in more severe plastic deformation around existing or fatigue-initiated flaws in the component. Such new material systems are encountered in automotive plain bearings where they provide a compromise between various design requirements such as load capacity, size and energy efficiency.

The complex crack growth patterns observed in multi-layer architectures has been attributed to the mismatch of mechanical properties between layers. Through-thickness cracks approaching a stiffer layer appeared to deflect along single-tip or bifurcated paths under service conditions. Similar behaviour has also been observed in multi-layered systems under cyclic three-point bending $[1,2]$ despite the absence of far-field mixed-mode loading conditions. The study of cracks subjected to large scale yielding (LSY) conditions requires the use of crack driving force (CDF) parameters, which should be valid beyond the scope of linear elastic fracture mechanics (LEFM). The $J$ integral [3] and crack tip opening displacement (CTOD) [4] have been used to evaluate the crack growth potential of stationary [5-7] and fatigue cracks [8] in diverse materials under elasto-plastic conditions.

Pioneering studies by Suresh et al [9] and Sugimura et al [10,11] investigated shielding and anti-shielding effects on the crack growth rate of plastically mismatched layers when the crack tip approached a stiffer or more compliant layer, respectively. Experimental and numerical 
studies by Joyce et al [1] on material systems consisting of steel and aluminium alloy layers under three-point bending corroborated previous shielding and anti-shielding observations and reported that, as the crack tip approaches a stiffer layer, CDF values increase with the deflection angle of bifurcated crack paths. Jiang et al [12] also observed shielding and anti-shielding trends in plastically mismatched steels subjected to four-point bending tests; they did not, however, report bifurcated or single-tip deflection as the crack tip approached a stiffer layer, attributing this effect to the presence of negative $T$ stress.

Crack deflection has been extensively studied in both brittle and ductile materials [13-16]. Bifurcation has also been widely studied in brittle materials [17-20] where shielding appears more consistently. It has however received less attention in multi-layered architectures composed of ductile materials [21-23]. Factors promoting bifurcation such as compressive residual stresses [18-20] and dynamic instability [24, 25] have been investigated leading to critical conditions for each particular case.

Various schemes have been introduced for extending and automatically predicting crack growth in two-dimensional fracture and fatigue analyses based on FE. Re-meshing crack extension schemes $[26,27]$ have been shown to be well suited for such tasks, which can be performed at a reasonable computational cost. Three-dimensional FE crack growth analyses showed $[28,29]$ that re-meshing schemes can be used efficiently on problems with complex geometries and loading conditions. Other schemes involving node release [30] and cohesive elements [31] were shown to improve computational efficiency in general terms and facilitate the inclusion of stress history but they suffer from a certain mesh bias. Recent developments [32] on 
the use of cohesive elements obviated the need of a pre-determined path, creating such elements where and when the maximum principal stress reaches the proportional limit.

Several criteria $[13,15-17]$ have been shown to predict crack deflection in agreement with experimental results under monotonic loading, small scale yielding and mode-I dominant, mixed-mode loading conditions. Of special interest is the difference observed between the propagation paths under monotonic and cyclic loading described by Mageed and Pandey [33] and Qian and Fatemi [34]. A uniformly consistent criterion for any loading condition has not been established and most of the applications of such criteria with FE analyses have been carried out using closed-form, near-tip solutions derived from elastic analyses. There is a prospect therefore for further investigation of deflected paths within the scope of elastic plastic fracture mechanics (EPFM) through numerical analysis.

As pointed out already, the study of the crack shielding observed in multi-layered architectures has been addressed previously by many authors. However, the consequences of shielding, that is, crack deflection and bifurcation, have not been investigated to a great depth. At the same time, the search of a rational criterion for determining the bifurcation point along a crack in ductile layers provides an opportunity for further development work.

The issues raised above are addressed in this paper. CDF evolution is analytically investigated for stationary and incremented cracks in multi-layer systems with mechanical properties mismatch that are subjected to loads causing large-scale yielding. The state of strain and stress around the crack tip is also studied to search for the conditions that promote crack deflection and 
bifurcation even under far-field pure mode I loading. The implementation of re-meshing schemes in FE crack extension modelling is preceded by the step-by-step determination of the deflected path followed by the crack using a validated deflection criterion.

\section{Methodology}

The study of propagating cracks in multi-layered systems under large scale yielding conditions is based on two-dimensional FE analyses and appropriate fracture mechanics concepts and methodology. Quasi-static simulations of crack growth are achieved by estimating the state of stress around the crack tip and the CDF parameter for straight and deflected crack paths.

This work is mainly concerned with crack deflection as a consequence of shielding; for this reason, various path deflection criteria are first assessed through two-dimensional FE simulations of and comparisons with the fracture and fatigue experiments carried out by Mageed and Pandey [33]. The crack shielding and deflection study itself is based on FE simulations of three-point bending tests and is carried out in two main stages. Initially, crack shielding and amplification is assessed for straight stationary cracks of variable length under pure mode-I loading that approach either stiffer or more compliant layers. This stage is similar to earlier investigations on shielding and amplification cited in the Introduction apart from the extent of plasticity and the use of CTOD as the CDF parameter. The second stage is concerned with the consequences of shielding and amplification and it addresses this issue in two ways. The first is concerned with the possibility of an initially straight crack under pure mode-I loading propagating along a single deflected path according to the adopted path deflection criterion; the second, with the conditions leading to the experimentally observed propagation of shielded cracks along a bifurcated path. 
The performed FE analyses are based on the general-purpose software ANSYS, version 10.0

[35]. Two-dimensional models were built using re-meshing schemes around straight or deflected propagating cracks. Parametric quarter-point elements [36] in spider-web configurations were found to be excessively distorted in preliminary simulations of three-point bending tests under high loads. For this reason, a blunted tip [37] modelled as a semi-circle of very small radius (around $0.1 \mu \mathrm{m}$ ), as shown in Fig. 1, was adopted for predicting reliably large crack tip deformations. The typical element size around the blunted tip was about $10 \mathrm{~nm}$, which can be compared to the overall dimensions of the models described in the subsequent sections to provide an idea of the degree of mesh refinement. Relevant stress and displacement values were read relative to a local polar frame of reference with origin at the centre of the semi-circle. True stress-true strain curves represent the plastic behaviour of the layers beyond the proportional limit. The compliance matrix is re-calculated after each convergence iteration in the solution process to account for the effect of large deformations observed in tests.

Compared to the $J$ integral, the CTOD is a less ambiguously defined and more easily computed CDF parameter for cracks propagating through layers of different elastic as well as plastic properties under LSY conditions. The CTOD is also theoretically better suited for characterising cyclic loading in the context of a fatigue crack propagation law. Another advantage of CTOD is its direct computation from FE displacement results. The CTOD was measured as the relative displacement of two nodes placed at the two ends of the blunted tip semi-circle, as shown in Fig. 1. A similar measurement has been reported from fatigue experiments on ductile materials [38, 39]. 


\section{Path Deflection Criteria}

The aim of the present study is crack growth assessment based on CTOD estimates, but also the investigation of crack deflection as a consequence of shielding due to the layered architecture. A criterion based on crack tip opening and shear displacements may not be the most appropriate for assessing the direction of crack deflection under LSY. It was thought, therefore, essential at this stage to examine the validity of the various crack deflection criteria that have been proposed in the literature through their implementation in FE analyses that simulate the mixed-mode fracture and fatigue experiments performed by Mageed and Pandey [33].

Mageed and Pandey tested 2024-T3 aluminium plates under tension with centre cracks at various angles to the loading axis to study the initial direction of the deflected crack under the generated mixed-mode loading conditions. Plates with initially straight 9-mm cracks at inclination angles $\beta$ of $30^{\circ}, 45^{\circ}, 60^{\circ}$ and $75^{\circ}$, measured from the axis normal to the loading direction, were tested under a cyclic tension ranging between 18 and $92.5 \mathrm{MPa}$. The specimen thickness, length and width were $1.27 \mathrm{~mm}, 250 \mathrm{~mm}$ and $180 \mathrm{~mm}$, respectively. The Young's modulus, Poisson's ratio, yield stress, ultimate stress and elongation to fracture of the material were reported as equal to $73.1 \mathrm{GPa}, 0.33,370 \mathrm{MPa}, 440 \mathrm{MPa}$ and $11 \%$, respectively. A true stress-true strain curve that fitted these data was constructed and entered into the FE model. A schematic view of the simulated arrangement is given in Fig. 2.

The applied crack deflection criteria were those based on the maximum tangential stress (MTS) [40], maximum tangential strain (MTSN) [16, 17], maximum principal stress (MPS) [41], maximum principal strain (MPSN) and crack tip displacement vector (CTDV) [15]. It should be 
noted that these criteria have had limited application within the context of EPFM under cyclic loading. Rather than relying on stress intensity factor calculations, the implementation of MTS and MTSN in this study was based on nodal FE results obtained around the blunted crack tip (see Fig. 1). The MPS, MPSN and CTDV criteria were implemented in a similar manner. The required parameters for each criterion were estimated at $5^{\circ}$ intervals. Quadratic interpolation through these values provided a more precise estimate of their maximum as well as the corresponding deflection angle $\alpha$.

The successful simulation of these experiments was based on modelling schemes identical to the ones described in Section 2, also used at later stages. Plane stress conditions were assumed due to the small thickness of the specimens. The FE predictions for the deflection angle $\alpha$ are presented together with the experimental measurements in Fig. 3 for the four inclination angles $\beta$ of the remote tensile stress of 92.5 MPa relative to the crack opening direction. Around a blunted crack tip, the tangential stresses and strains coincide with their respective principal values; it was therefore expected that MPSN and MPS would give results identical to those obtained using MTSN and MTS, respectively.

The deflected direction of propagation under applied tension at $30^{\circ}$ to the crack opening direction, which corresponds to the most mode-I dominant loading conditions considered, is consistently predicted by CTDV and MTSN criteria and these predictions are in good agreement with the respective experimental result. The agreement of MTSN predictions with experimental results is slightly reduced with increasing crack inclination angle $\beta$ while those based on CTDV follow an opposite trend. The MTS criterion appears to underestimate initially the deflection 
angle but its predictions become more consistent with those of MTSN as the inclination angle increases. The FE analyses were repeated under the minimum applied tensile stress of $18 \mathrm{MPa}$ and, in the case of MTSN and CTDV, the respective deflection angle predictions were slightly greater (by around 4\%) than those obtained under maximum load. The MTS criterion led to identical results under both load levels.

The main conclusion from the results above is that, under conditions of extended plasticity, a strain-based approach provides estimates of crack deflection angles more consistent with experimental evidence than those obtained using a stress-based criterion. For this reason, the MTSN criterion is used in subsequent stages of this study in order to assess the direction of propagation. It is expected that crack propagation would occur along a direction bounded by the deflection angle estimates obtained at the maximum and minimum load. Since however, the difference between these two values was found to be small, the analysis per crack increment will be performed only under maximum load thus saving computational effort and time. The application of MTSN criterion will be monitored so that it is limited to mode-I dominant, mixedmode loading for which the crack deflection estimates were found to be most reliable.

\section{Straight cracks}

The evolution of CTOD for straight cracks of variable length as the crack tip approaches a stiffer or more compliant layer is studied in bi-layer and tri-layer architectures under three-point bending. The applied load was varied to cause different extents of plasticity in the analysed material system. In parallel to the investigation of conditions around the crack tip that may cause 
path deflection or bifurcation, initial estimates of the most likely direction of propagation were also obtained.

The tri-layer architecture of a recently tested flat bearing strip [42] was analysed. The test arrangement is schematically drawn in Fig. 4. The specimen consisted of a backing steel layer of thickness $t_{b}=1.80 \mathrm{~mm}$, a lining aluminium alloy layer of thickness $t_{\mathrm{L} 1}=0.38 \mathrm{~mm}$ and an aluminium foil interlayer of thickness $t_{i}=0.04 \mathrm{~mm}$. The bi-layer model was a hypothetical one consisting of the same backing layer as that of the tri-layer model and an aluminium alloy lining layer of thickness $t_{\mathrm{L} 2}=t_{\mathrm{L} 1}+t_{i}=0.42 \mathrm{~mm}$. The strip had a width $w=19.5 \mathrm{~mm}$ and length between supports $L=40 \mathrm{~mm}$. Two-dimensional models assuming plane strain conditions were developed in ANSYS using second order elements and a multi-linear hardening material model. A typical FE mesh comprised around 15,000 nodes; the degree of refinement around the crack tip is clearly illustrated in Fig. 1. Based on experimental observations as well as preliminary numerical tests, LSY conditions predominate at the higher range of the applied loads; therefore the mechanical properties of each layer material are entered in the form of the true stress-true strain curves of Fig. 5. The Young's modulus $E$, Poisson's ratio $v$, yield stress $\sigma_{Y}$, ultimate stress $\sigma_{u}$ and hardening exponent $n$ associated with these curves are listed in Table 1.

The variation of normalised CTOD with crack length in both the bi-layer and tri-layer systems under 3 levels of loading is shown in Fig. 6. In this as well as all subsequent graphs, the CTOD results are plotted against the ratio of the crack length $a$ to the lining thickness $t_{\mathrm{L} 2}$ of the bi-layer system. The shielding and amplification trends observed in Fig. 6 are consistent with those reported in similar previous investigations based on $J$-integral estimates of systems with 
principally plastic [9] but also elastic $[1,43]$ mismatch. In the bi-layer architecture, the shielding effect is indicated by the decrease in CTOD as the crack approaches the stiffer steel layer. In the tri-layer architecture, the amplification effect is noted when the crack approaches the more compliant interlayer, and the shielding effect when the crack approaches the stiffer backing. The application of higher loads and subsequent spread of plasticity led to greater mismatches between layers, which caused increased CTOD gradients and therefore more pronounced shielding and amplification at lower crack lengths [43]; in contrast, the spread of plasticity into the backing layer of the bi-layer strip at the highest load reduced the mismatch between the layers and, as a consequence, shifted the CTOD maximum closer to the aluminium/steel interface.

The evaluated crack tip tangential strains (CTTS) showed different patterns depending on the applied load, crack length, layer in which the crack tip is located and architecture analysed. At crack lengths below $0.45 t_{\mathrm{L} 2}$ and $0.54 t_{\mathrm{L} 2}$ in the bi-layer and tri-layer strips, respectively, the CTTS had a clear maximum (MTSN) when plotted against the angular position around the blunted tip; this clearly indicated the normal to the interface as the preferred direction of propagation as observed experimentally. The dependence of CTTS curves on crack length is shown in Fig. 7 for the bi-layer strip under maximum load $P=800 \mathrm{~N}$. The maximum tangential strain is clearly identified for low crack lengths but as the crack approaches the backing layer, the existence and location of a CTTS maximum becomes less obvious. This is the first indication that the shielded crack may follow a deflected or bifurcated path. 
The CTOD as CDF parameter is thus shown to be well suited for investigating crack shielding and amplification in multi-layered systems under LSY conditions. The results obtained with CTOD are consistent with those previously published [9-11, 44], despite using a different CDF parameter. The results for CTTS are also interesting since they hint at possible crack behaviour as the crack tip approaches a layer interface.

\section{Deflected cracks}

The development of a suitable criterion to forecast the potential for crack bifurcation in layered ductile materials remains an important analysis objective. Such criteria were proposed by Ho et al [45] and extended by Lugovy et al [18] for brittle multi-layered systems under significant residual stresses. They were based on the material stiffness, Poisson's ratios and critical stress intensity factors, that is, on concepts that have no relevance to the LSY problems under consideration. As indicated in the previous section, the application of a MTSN-based deflection criterion is not only reliable but also far more appropriate.

\subsection{Single deflected crack path}

Shielding and amplification effects as the tip of a straight crack approaches either a stiffer or a more compliant layer have been previously identified through the evolution of CDF adopted by various investigators. However, the consequences of shielding, considering the possibility of path deflection, have not been studied to great depth. Deflected crack propagation is here studied in both the bi-layer and tri-layer architectures described in Section 4. The initial flaw was again placed at the centre of the specimen surface as observed experimentally under three-point bending (see Fig. 4). Two different load magnitudes, $P=200 \mathrm{~N}$ and $P=800 \mathrm{~N}$, were applied causing moderate and large-scale yielding, respectively. The inclusion of path deflection in the modelling of the crack extension process was carried out through a re-meshing scheme linked to 
the estimated evolution of the crack driving force and the maximum tangential strain criterion [16]. This process creates short crack extensions along the predicted direction according to the near-tip state of stress at the previous step; the re-meshing scheme described by Colombo and Giglio [27] was adopted. The concatenation of short crack extensions forms the path along which the values of the crack driving force are obtained.

Results from the tri-layer architecture indicated distinct trends of crack propagation in different layers. At both load levels applied, the crack growth direction within the lining remained unaffected as the crack tip approached the more compliant interlayer. CTOD increased with crack extension as previously observed with stationary straight cracks (see Fig. 6). The curvature of the CTTS curves became slightly smaller at maximum as the tip approached the compliant interlayer, especially at the upper load level. However, the maximum CTTS value (MTSN) could still be identified as the one forecasting a straight crack path normal to the interface.

As soon as the tip penetrated into the interlayer, it was not possible to identify a single maximum along the obtained CTTS curves; the extent of this curve flattening depended on the applied load. This meant that almost identical CTTS values emerged symmetrically located with respect to the original direction of propagation corresponding to polar angle $\theta=0^{\circ}$. Despite the small variation of CTTS within a wide range of polar angles, an MTSN value was numerically identified at $\theta=16.4^{\circ}$. It was assumed that a small deviation from loading or geometric symmetry would cause the crack to deflect at this angle, which was thus used to define the orientation of the introduced short kink. This initial extension was followed by smaller 
subsequent deflections so that the crack path approached asymptotically the interlayer-backing interface as shown in Fig. 8. The CTTS variation along the blunted crack tip at various deflected crack extensions is plotted in Fig. 9. It is interesting to notice that the MTSN becomes progressively more distinguishable and uniquely identifiable immediately after the first deflection.

The CTOD estimates obtained from the straight stationary and incremented deflected cracks as well as the accumulated deflection angle in the interlayer are presented in Fig. 10 for $P=200$ $\mathrm{N}$. This figure clearly shows that the predicted CDF is greater in the deflected than in the straight path, a result consistent with the principle of crack propagation in the direction of least resistance, corresponding to maximum CDF [46]. Similar path deflections in the interlayer were predicted by the analysis performed under $P=800 \mathrm{~N}$. Apart from the expected increase in CDF estimates, the main difference was a more pronounced initial deflection.

Crack deflection in the bi-layer system was found to be influenced to a greater extent by the intensity of the applied load. For $P=200 \mathrm{~N}$, initial $1^{\circ}$-deflections appeared very close to the steel layer (at $a=0.95 t_{\mathrm{L} 2}$ ); in contrast, an equivalent deflection appeared at $a=0.61 t_{\mathrm{L} 2}$ for $P=800 \mathrm{~N}$. The CDF estimates in the bi-layer system were also slightly greater along the deflected than the straight path at both load levels, in qualitative agreement with the findings from the tri-layer architecture.

Previous works on shielding and anti-shielding in layered architectures have usually explained these phenomena in terms of differences between the materials' yield stress. However, the yield 
stress may be considered as reflecting only partly material influence since it is not fully responsible for the post-yield deformation and the state of stress. An additional factor would be the difference between the materials' strain hardening rate represented by the gradient of the $\sigma-\varepsilon$ curves since this is also directly related to the material resistance to deformation. Such a relation is simple when elastic analyses are carried out. Nevertheless, elasto-plastic analyses result in a widely ranging gradient depending on the stress concentration patterns developing around the crack tip.

It has been shown that a single-tip deflected crack path is possible despite the absence of farfield mixed-mode loading. It is worth noting that the maximum CTTS value (MTSN), according to which crack path deflection is predicted, is essentially reached over a wide range of direction angles, depending on crack tip proximity to the interface and the intensity of the applied load. This indicates a high sensitivity of the propagation process to heterogeneities in the material that are likely to disturb the perfect symmetry of the problem and thus initiate deflection. This sensitivity is reduced as the deflected crack propagates away from the plane of symmetry gradually reaching a more stable state.

The assessment of crack path sensitivity to a perturbation is limited by the uncertainty about which side to the symmetry plane deflection would occur on but it is expected that the dominance of the far field loading conditions would lead to consistent outcomes whatever the direction of an introduced perturbation. This hypothesis was tested by introducing an arbitrary initial deflection at $30^{\circ}$ to the straight crack path both in the lining and the interlayer. In both cases, the perturbed crack path gradually approached and then, after 3 or 4 cycles, followed the 
deflected crack path predicted from an initial kink angle corresponding to maximum CTTS. The quick re-alignment of the path showed the predominance of far-field loading and specimen properties over the effect of a local perturbation in this particular example.

Crack deflection has been linked to service life extension through the lengthening of the crack path and the concomitant reduction of CDF along the deflected path. Material homogeneity has been assumed in this work focusing on the mismatch between layers in the architecture. Material microstructure has been overlooked but this heterogeneity may lead to a more tortuous crack path and thus extend the crack length further. Such a combination of global and local deflections has been observed in flat strip specimens under three-point bending tests [2].

\subsection{Bifurcated crack path}

Crack path bifurcation has been observed experimentally [2, 21-23], it is therefore important that this possibility be investigated analytically as well. The aim of numerical analysis at this stage would be to show that conditions favouring bifurcation may develop in bi-layer and trilayer systems under fatigue loading. The location of the bifurcation point and subsequent crack growth are complex materials issues that require further study, beyond the scope of this paper.

Crack bifurcation in automotive bearing systems has been linked to shielding as the crack approaches a stiffer layer [2]. Based on such observations, bifurcation was investigated in the lining of the bi-layer system and the interlayer of the tri-layer system. Conditions for bifurcation initiation were studied by focusing on the effect of the straight crack length as well as incremental kink angle and length on the CDF at the onset of bifurcation. Taking advantage of 
the symmetry of the problem, only half of the strip specimen was modelled and analysed as shown in Fig. 11.

In order to assess the initial bifurcation conditions, a parametric study was performed involving bifurcated crack kinks of length between $1 \%$ and $5 \%$ of the thickness of the layer containing the crack tip. The results from both layered systems showed that for any given location of the bifurcation point relative to the interface there exists a kink angle that maximises the CDF, which can be interpreted as the most likely deflection direction. The variation of kink length within the limits mentioned above did not have any significant effect on the predicted deflection angle. The CDF estimates obtained in the case of the tri-layer architecture are shown in Fig. 12. A gradual shift of the maximum to greater kink angles can be observed as the bifurcation point approaches the interface. The last case analysed, corresponding to a straight crack length of $0.993 t_{\mathrm{L} 2}$, results in a steep increment of the predicted deflection angle. This confirms the strong shielding effect of the stiffer and harder steel layer, which forces the crack to propagate parallel to rather than through it. In contrast, there is no emphatic propensity for the crack tip to deflect within the lining as indicated by the CTOD curve obtained for $a=0.8 t_{\mathrm{L} 2}$. In the case of the bi-layer model, the possible initial bifurcation angle can be obtained from Fig. 13, which shows that it increases gradually from $0^{\circ}$ to around $45^{\circ}$ for straight crack lengths ranging from $0.3 t_{\mathrm{L} 2}$ to $0.95 t_{\mathrm{L} 2}$, respectively.

As noted with the single path deflection presented in the previous section, the location of the bifurcation point also influenced the magnitude of CDF estimates showing shielding effects associated with proximity to a stiffer layer. Due to the thinness of the interlayer, shielding in the 
tri-layer architecture was observed as soon as the bifurcation point crossed into the interlayer while shielding in the bi-layer architecture became apparent only after the straight crack length grew above $0.7 t_{\mathrm{L} 2}$. The variation of the CTTS estimates around the blunted crack tip of straight stationary cracks also indicated a trend for crack bifurcation since they displayed maxima away from the straight crack direction. The kink angle corresponding to such a maximum can be selected as a possible initial deflection angle for the following rough analysis of bifurcated branch growth.

Figs. 12 and 13 show that the CDF predictions for different initial crack bifurcations depend greatly on the kink angle, showing a clear maximum value. The kink angle corresponding to this maximum value rises as the bifurcation point approaches a stiffer layer. The predicted direction of the bifurcated path depends on the extent of property mismatch and the proximity of the crack tip to the layer interface, as reported previously by Joyce et al [1]. In this earlier work, the kink angle corresponding to the maximum $\mathrm{CDF}$, represented by the $J$-integral, reached the value of $90^{\circ}$. Using MTSN as the deflection criterion did not lead to path deflections of such a magnitude. This may be due to an inherent limitation of the MTSN criterion to predict significant changes in path direction; its suitability needs, therefore, to be investigated further. It is also worth mentioning that the steel backing was assumed elastic by Joyce et al, while here plastic deformation was allowed to develop when the load reached a certain value. The proximity of MTSN and MTS predictions for higher mode mixing, manifested in Fig. 3, points to the work of Li et al [47], who discussed the limitations of the MTS under dominant mode-II loading and proposed that the maximum shear stress may be a more appropriate criterion for crack growth under such a high load mixity condition. 
The computation of the CTOD is an area where improvements in the adopted methodology can be made since large shearing deformations distort the crack tip elements significantly leading to underestimation of the actual CTOD value. A systematic use of multiple nodes along the crack faces may be a feasible option for obtaining more reliable estimates of crack opening and sliding displacements under mode II-dominant loading conditions.

The extension of the analysis to the prediction of bifurcated crack growth requires careful examination of the material and loading conditions encouraging such an event. While single tip deflection under mode I far-field loading can be micro-structurally induced [48], crack branching is possible only if available crack extension energy momentarily far exceeds material resistance, as happens, for instance, in the case of overloading [44]. This explains the reported bifurcation events within the interlayer of tri-layer systems during previous experimental investigations on the fatigue performance of bearings [2]. The sharp increase in CDF noted in Fig. 6 as the crack approaches the lining-interlayer boundary is followed by a sudden drop in material resistance as soon as the crack crosses that boundary. It is therefore important for a numerical model of bifurcated crack growth to account for such material changes as well as the possibility of subsequent asymmetric crack growth.

\section{Discussion and Conclusions}

The present study does not include the effect of residual stresses that arise from manufacturing processes, cycling loading, interacting micro-cracks, or crack closure during crack growth. It has been shown that the use of CTOD as CDF is well suited to the study of crack growth and deflection under LSY conditions leading to trends consistent with those of previously published investigations on shielding and amplification in multi-layered systems. It 
has been implicitly assumed that the adopted CDF parameter is proportional to crack growth rate as suggested by Tomkins [49].

The adopted methodology for studying in detail the stress and strain fields around the crack tip and their interaction with the layers interfaces appears to be an effective tool for modelling growth of deflected cracks under LSY conditions. However, the complexity of mesh generation and the large number of degrees of freedom required inhibit the applicability of this methodology to the analysis of problems with multiple cracks; the need for advanced modelling skills to generate automated crack extension steps and the considerable computational cost involved has led to the application of alternative techniques such as meshless methods and X-FEM. These methods appear to be particularly convenient for two-dimensional analyses within LEFM. Their applicability to large deformation problems in the context of EPFM and to three-dimensional analyses has been tested to a certain degree.

The estimated path for propagating deflected cracks is consistent with experimental results predicting path alignment with the interface; this gives confidence in the validity of the deflection criterion and its implementation. The inclusion of the elasto-plastic behaviour of the materials in the FE modelling has a significant effect on the results of the analysed problem due to the extent of plasticity observed around the crack tip and the kinematic constraint imposed by the stiff backing layer.

The stationary and propagating straight crack analyses show that the CTTS patterns may be related to single-tip deflection and bifurcation events caused by the property mismatch between 
layers and such events may occur despite far-field pure mode-I loading conditions. The observed spread of maximum CTTS values indicates a tendency of the crack to deviate from its original straight path reducing the influence of the symmetric far field loading conditions and increasing the effect of local random material microstructure. A more rigorous micro-mechanical analysis is necessary to support this hypothesis and provide definitive conclusions; however the results of this investigation can be considered a sound basis for further work.

The analysis of deflected cracks showed that such a scenario could occur in flat strip specimens under three-point bending tests. Microstructural features and mechanisms were not included in these analyses directly. The presented methodology addresses these issues from a continuum perspective and can be applied to the determination of the service life of a given component only when it is, at least, combined with both the crack growth law and the crack growth resistance fitted to reliable experimental data.

\section{Acknowledgements}

The authors gratefully acknowledge the support for this work provided by CONACYT (Mexican National Council of Science and Technology) and the University of Southampton.

\section{References}

[1] Joyce MR, Reed PAS, Syngellakis S. Numerical modelling of crack shielding and deflection in a multi-layered material system. Mater Sci Eng A-Struct Mater Prop Microstruct Process. 2003;342(1-2):11-22. 
[2] Joyce MR, Syngellakis S, Reed PAS. Fatigue crack initiation and early growth in a multiphase Al alloy included in a multilayer material system. Mater Sci Techol. 2004;20(1):4756.

[3] Rice JR. A path independent integral and the approximate analysis of strain concentration by notches and cracks. J Appl Mech-Trans ASME. 1968;35:379-386.

[4] Wells AA. Unstable crack propagation in metals: cleavage and fast fracture. Proceedings of the Crack Propagation Symposium. Cranfield, UK; 1961.

[5] May GB, Kobayashi AS. Plane stress stable crack growth and J-integral/HRR field. Int J Solids Struct. 1995;32(6-7):857-881.

[6] Newman JJC, James MA, Zerbst U. A review of the CTOA/CTOD fracture criterion. Eng Fract Mech. 2003;70(3-4):371-385.

[7] Ostby E, Thaulow C, Nyhus B. A new approach to ductile tearing assessment of pipelines under large-scale yielding. Int J Pressure Vessels Pip. 2007;84(6):337-348.

[8] Dowling NE, Begley JA. Fatigue crack growth during gross plasticity and the J integral. In: Rice JR, Paris PC, editors. Mechanics of Crack Growth, STP 590. Philadelphia: American Society for Testing and Materials, 1976. p. 82-103.

[9] Suresh S, Sugimura Y, Tschegg EK. Growth of a fatigue crack approaching a perpendicularly oriented bimaterial interface. Scripta Metall Mater. 1992;27:1189-1194. [10] Sugimura Y, Lim PG, Shih CF, Suresh S. Fracture normal to a bimaterial interface: Effects of plasticity on crack-tip shielding and amplification. Acta Metall Mater. 1995;43(3):1157-1169.

[11] Sugimura Y, Grondin L, Suresh S. Fatigue crack growth at arbitrary angles to bimaterial interfaces. Scripta Metall Mater. 1995;33(12):2007-2012. 
[12] Jiang F, Deng ZL, Zhao K, Sun J. Fatigue crack propagation normal to a plasticity mismatched bimaterial interface. Mater Sci Eng A-Struct Mater Prop Microstruct Process. $2003 ; 356(1-2): 258-266$.

[13] Sih GC. Strain energy density factor applied to mixed mode crack problems. Int J Fract. $1974 ; 10: 305-321$

[14] Sih GC, Barthelemy BM. Mixed mode fatigue crack growth predictions. Eng Fract Mech. $1980 ; 13(3): 439-451$

[15] Li C. Vector CTD criterion applied to mixed mode fatigue crack growth. Fatigue Fract Eng Mater Struct. 1989;12(1):59-65.

[16] Chambers AC, Hyde TH, Webster JJ. Mixed mode fatigue crack growth at $550{ }^{\circ} \mathrm{C}$ under plane stress conditions in Jethete M152. Eng Fract Mech. 1991;39(3):603-619.

[17] Maiti SK, Smith RA. Criteria for brittle fracture in biaxial tension. Eng Fract Mech. $1984 ; 19(5): 793-804$

[18] Lugovy M, Orlovskaya N, Slyunyayev V, Gogotsi G, Kubler J, Sanchez-Herencia AJ. Crack bifurcation features in laminar specimens with fixed total thickness. Compos Sci Technol. $2002 ; 62(6): 819-830$.

[19] Pontin MG, Lange FF. Crack bifurcation at the surface of laminar ceramics that exhibit a threshold strength. J Am Ceram Soc. 2005;88(5):1315-1317.

[20] Hbaieb K, McMeeking RM, Lange FF. Crack bifurcation in laminar ceramics having large compressive stress. Int J Solids Struct. 2007;44(10):3328-3343.

[21] Keesecker AL, Davila CG, Johnson ER, Starnes JH. Crack path bifurcation at a tear strap in a pressurized shell. Comput Struct. 2003;81(16):1633-1642. 
[22] Suzuki S, Sakaue K, Iwanaga K. Measurement of energy release rate and energy flux of rapidly bifurcating crack in Homalite 100 and Araldite B by high-speed holographic microscopy. J Mech Phys Solids. 2007;55(7):1487-1512.

[23] Pippan R, Flechsig K, Riemelmoser FO. Fatigue crack propagation behavior in the vicinity of an interface between materials with different yield stresses. Mater Sci Eng A-Struct Mater Prop Microstruct Process. 2000;283(1-2):225-233.

[24] Murphy N, Ali M, Ivankovic A. Dynamic crack bifurcation in PMMA. Eng Fract Mech. 2006;73(16):2569-2587.

[25] Katzav E, Adda-Bedia M, Arias R. Theory of dynamic crack branching in brittle materials. Int J Fract. 2007;143(3):245-271.

[26] Wawrzynek PA, Ingraffea AR. An interactive approach to local remeshing around a propagating crack. Finite Elem Anal Des. 1989;5(1):87-96.

[27] Colombo D, Giglio M. A methodology for automatic crack propagation modelling in planar and shell FE models. Eng Fract Mech. 2006;73(4):490-504.

[28] Ural A, Heber G, Wawrzynek PA, Ingraffea AR, Lewicki DG, Neto JBC. Threedimensional, parallel, finite element simulation of fatigue crack growth in a spiral bevel pinion gear. Eng Fract Mech. 2005;72(8):1148-1170.

[29] Barlow KW, Chandra R. Fatigue crack propagation simulation in an aircraft engine fan blade attachment. Int J Fatigue. 2005;27(10-12):1661-1668.

[30] Heper R, Vardar O. Elastic-plastic material response of fatigue crack surface profiles due to overload interactions. Int J Fatigue. 2003;25(9-11):801-810.

[31] Li W, Siegmund T. An analysis of crack growth in thin-sheet metal via a cohesive zone model. Eng Fract Mech. 2002;69(18):2073-2093. 
[32] Borst Rd, Remmers JJC, Needleman A. Mesh-independent discrete numerical representations of cohesive-zone models. Eng Fract Mech. 2006;73(2):160-177.

[33] Mageed AAM, Pandey RK. Mixed mode crack growth under static and cyclic loading in Al alloy sheets. Eng Fract Mech. 1991;40(2):371-375.

[34] Qian J, Fatemi A. Mixed mode fatigue crack growth: A literature survey. Eng Fract Mech. 1996;55(6):969-990.

[35] Ansys. Ansys reference manual. 2005.

[36] Barsoum R. Triangular quarter point elements as elastic and perfectly plastic crack tip elements. Int J Numer Methods Eng. 1977;11: 85-98.

[37] McMeeking RM, Parks DM. On criteria for J-dominance of crack-tip fields in large scale yielding. In: Landes JD, Begley JA, Clarke GA, editors. Elastic-Plastic Fracture, ASTM STP 668: Americal Society for Testing and Materials, 1979. p. 175-194.

[38] Pardoen T, Delannay F. A method for the metallographical measurement of the CTOD at cracking initiation and the role of reverse plasticity on unloading. Eng Fract Mech. 2000;65(4):455-466.

[39] Laird C, Smith GC. Crack propagation in high stress fatigue. Philos Mag. 1962;8:847857.

[40] Erdogan F, Sih GC. On the crack extension in plates under plane loading and transverse shear. J Basic Eng-Trans ASME. 1963;85:519-527.

[41] Iida S, Kobayashi A. Crack-propagation rate in 7075-T6 plates under cyclic tensile and transverse shear loadings. J Basic Eng-Trans ASME. 1969:764-769.

[42] Ali MS, Reed PAS, Syngellakis S, Moffat A, Perrin C. Microstructural factors affecting fatigue initiation in various Al based bearing alloys. Mater Sci Forum. 2006;519-521:1071-1076. 
[43] Predan J, Gubeljak N, Kolednik O. On the local variation of the crack driving force in a double mismatched weld. Eng Fract Mech. 2007;74(11):1739-1757.

[44] Suresh S. Micromechanisms of Fatigue Crack Growth Retardation Following Overloads. Eng Fract Mech. 1983;18(577-593).

[45] Ho S, Hillman C, Lange FF, Suo Z. Surface cracking in layers under biaxial, residual compressive stress. J Am Ceram Soc. 1995;78(9):2353-2359.

[46] Hussain MA, Pu SL, Underwood J. Strain energy release rate for a crack under combined mode I and mode II. In: Irwin GR, editor. Fracture Analysis: Proceedings of the 1973 National Symposium on Fracture Mechanics, Part II: ASTM STP 560, 1974. p. 2-28.

[47] Li J, Zhang X-B, Recho N. J-Mp based criteria for bifurcation assessment of a crack in elastic-plastic materials under mixed mode I-II loading. Eng Fract Mech. 2004;71(3):329-343. [48] Suresh S. Fatigue of Materials. Cambridge University Press, 1998.

[49] Tomkins B. Fatigue crack propagation - an analysis. Philos Mag. 1968;18(155):1041 1066. 


\section{Tables}

Table 1 Material properties for the three layers of the flat strip bearing system

\begin{tabular}{|c|c|c|c|c|c|}
\hline Layer & $\begin{array}{c}E \\
(\mathrm{GPa})\end{array}$ & $v$ & $\sigma_{Y}$ & $\sigma_{u}$ & $n$ \\
& & & $(\mathrm{MPa})$ & $(\mathrm{MPa})$ & \\
\hline Lining & 70 & 0.33 & 53 & 166 & 0.238 \\
\hline Interlayer & 67 & 0.33 & 35 & 78 & 0.19 \\
\hline Steel & 200 & 0.3 & 460 & 512 & 0.058 \\
\hline
\end{tabular}




\section{Figure captions}

Figure 1. Finite element model of blunted crack tip before and after deformation.

Figure 2. Schematic of the model simulating test arrangements by Mageed and Pandey [33].

Figure 3. Crack deflection predictions with corresponding fatigue test results [33] at 92.5 MPa.

Figure 4. Three-point bending and test specimen configuration.

Figure 5. True stress-true strain curves for the three-point bending model.

Figure 6. CTOD evolution with crack length in bi-layer and tri-layer systems.

Figure 7. Tangential strain variation around the crack tip at various crack lengths in the bi-layer system.

Figure 8. Predicted crack path within the soft interlayer of the tri-layer system.

Figure 9. Evolution of CTTS curves with crack extension steps.

Figure 10. Comparison of CDF(CTOD) evolution along straight and deflected paths in the tri-layer system under $P=200 \mathrm{~N}$.

Figure 11. Model with bifurcated crack with relevant notation.

Figure 12. Variation of CDF (normalised CTOD) with kink angle in the tri-layer architecture.

Figure 13. Variation of CDF (normalised CTOD) with kink angle in the bi-layer architecture. 
Figure 1

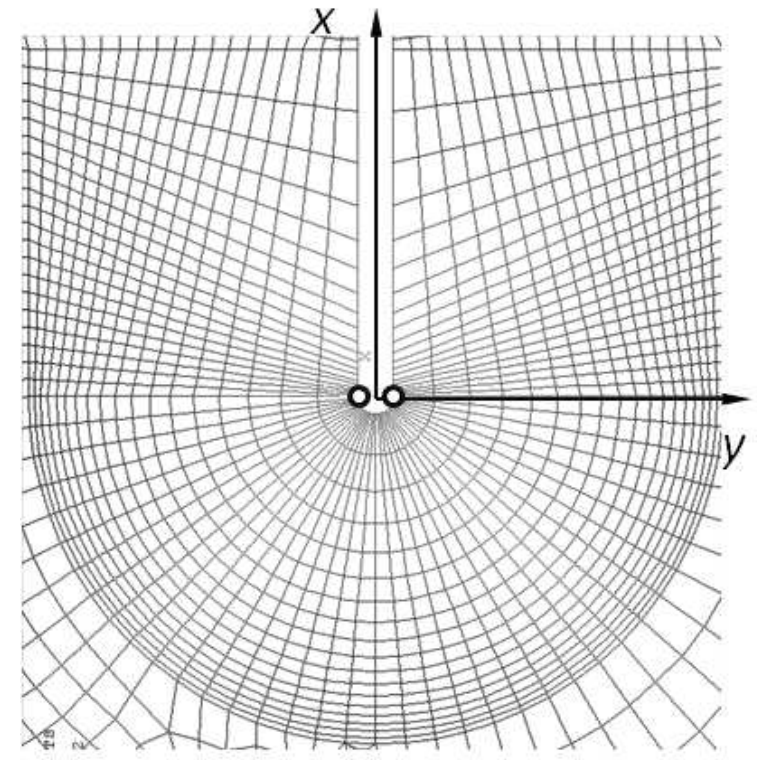

(a)

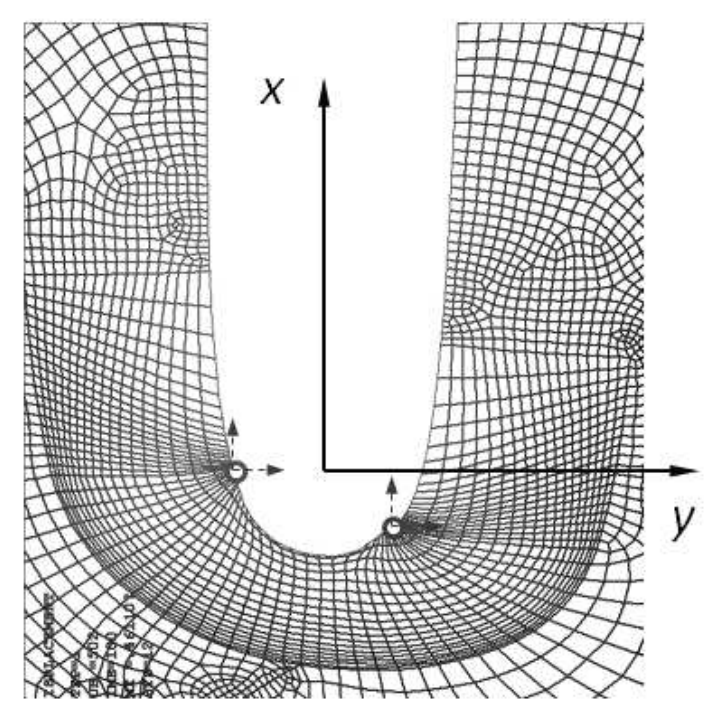

(b) 
Figure 2

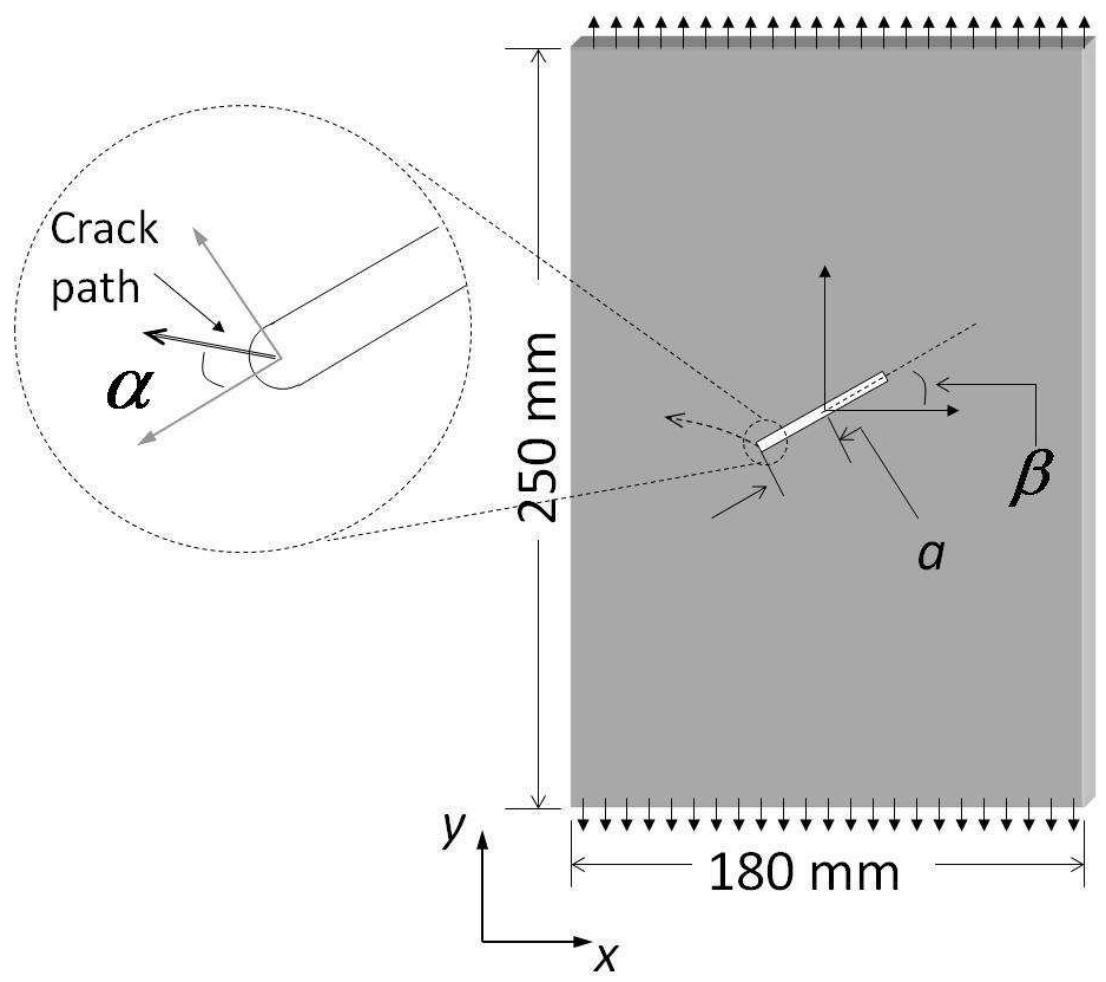


Figure 3

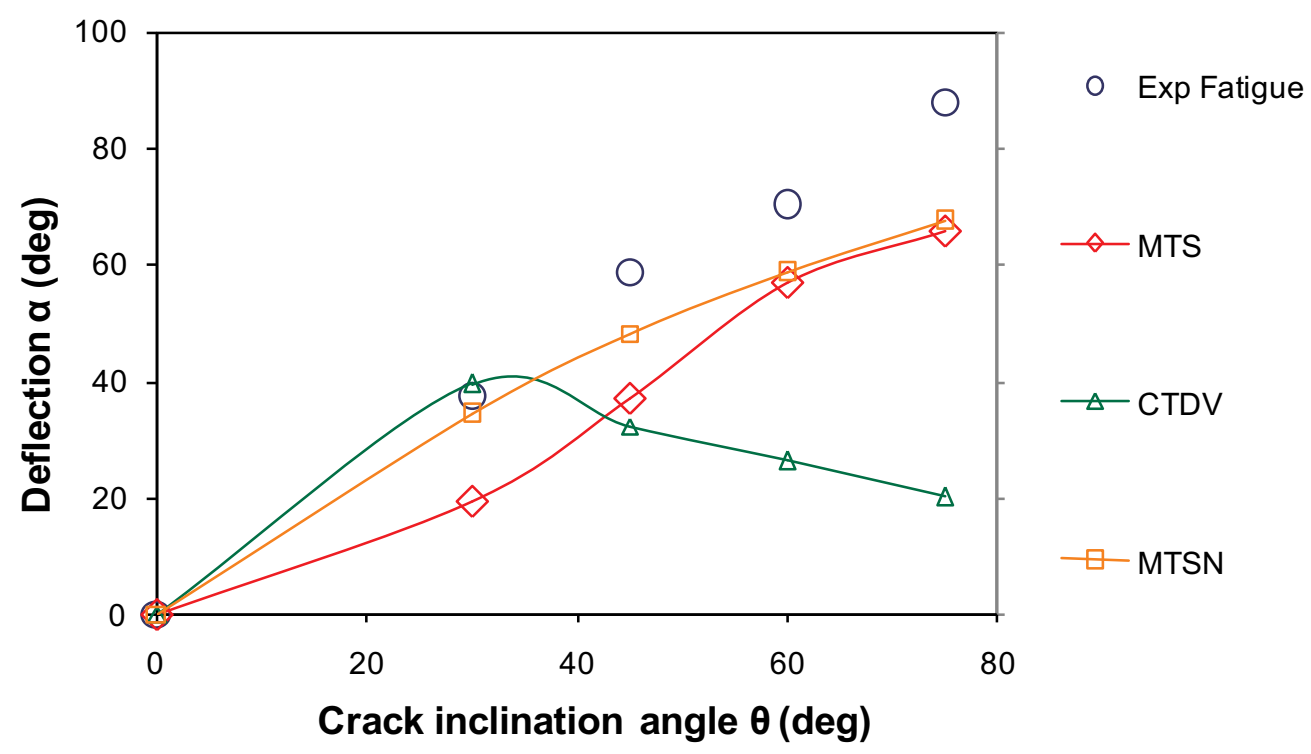


Figure 4

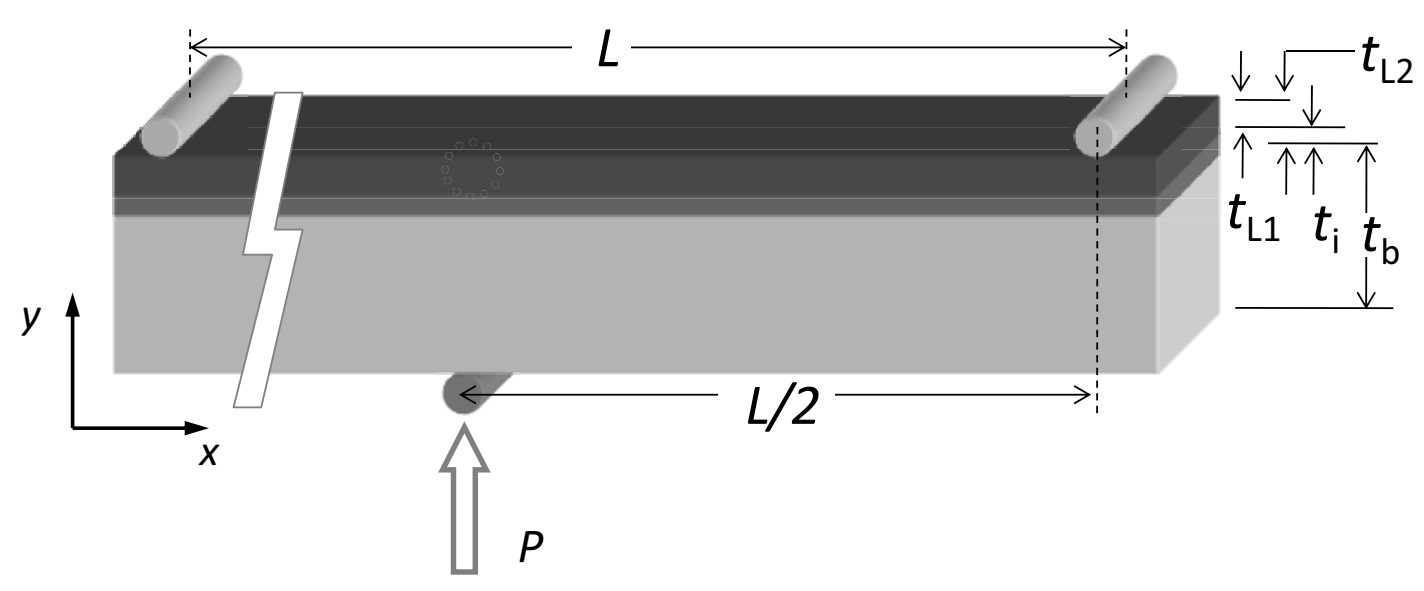


Figure 5

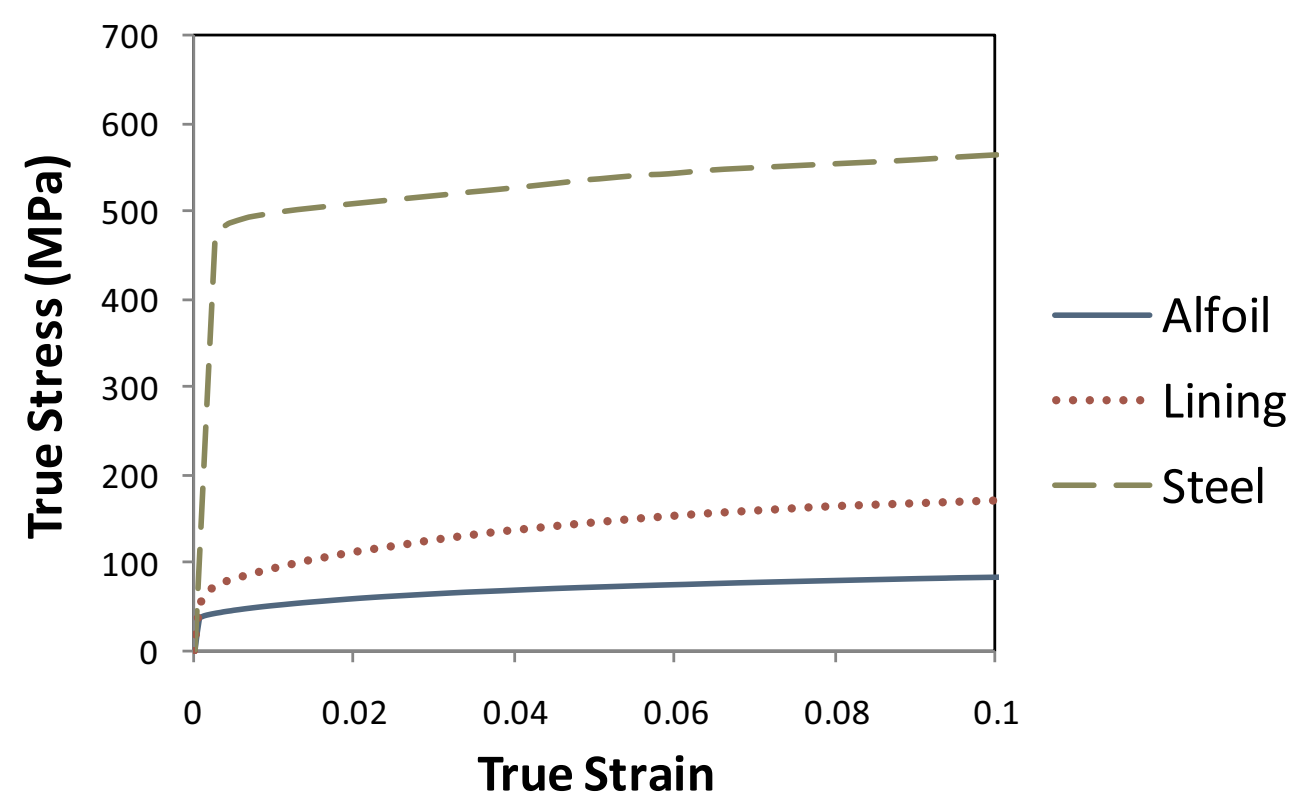


Figure 6
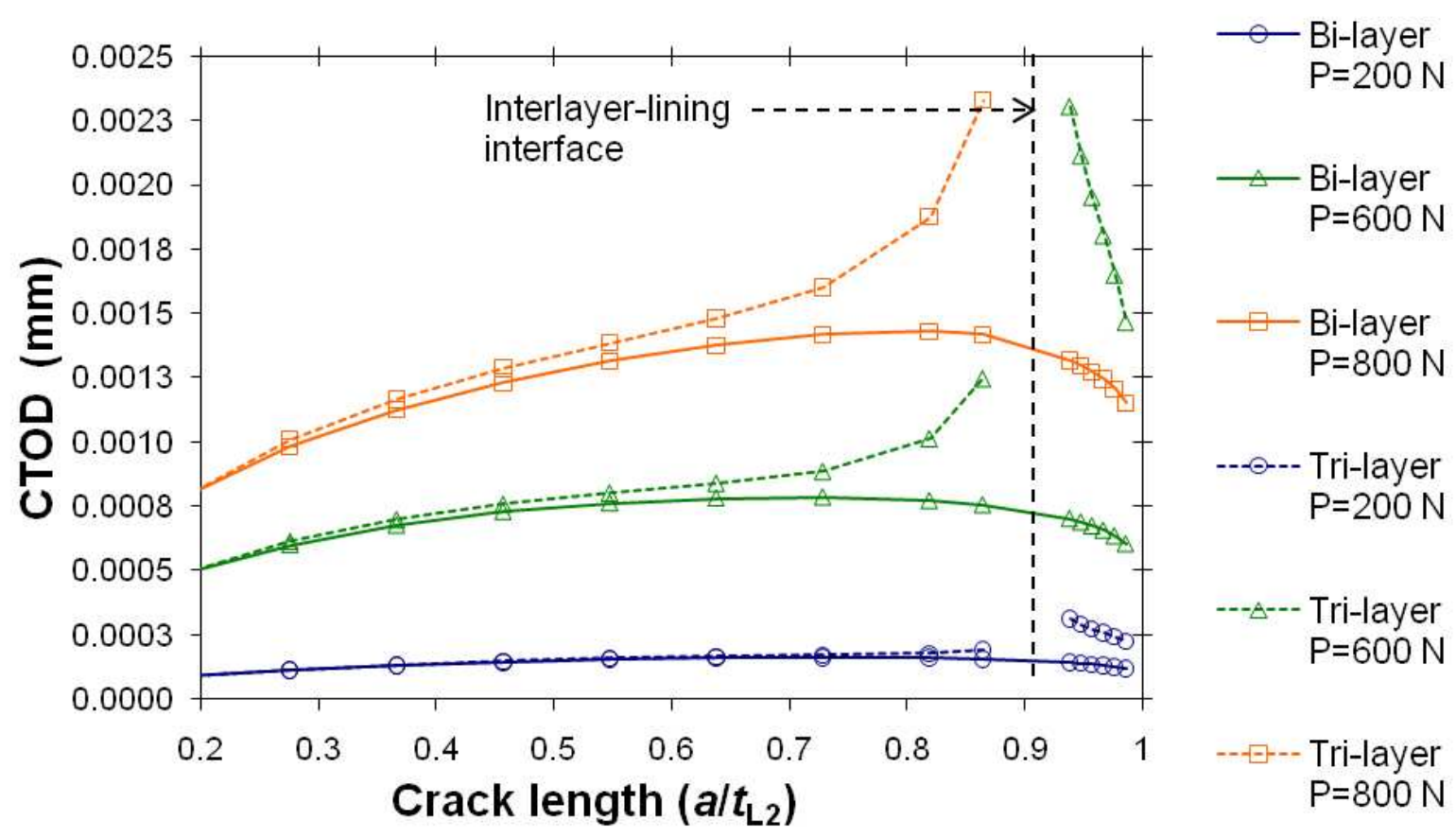
Figure 7

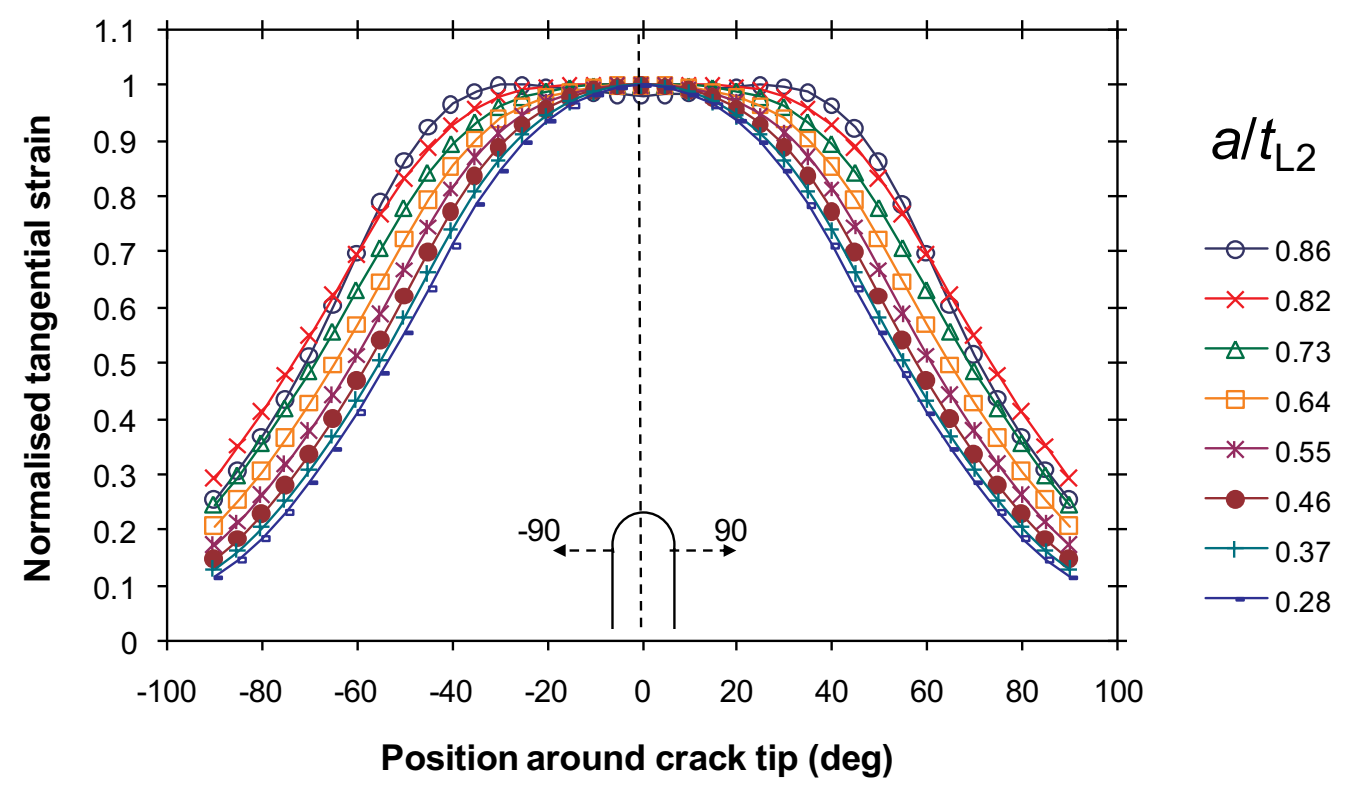


Figure 8

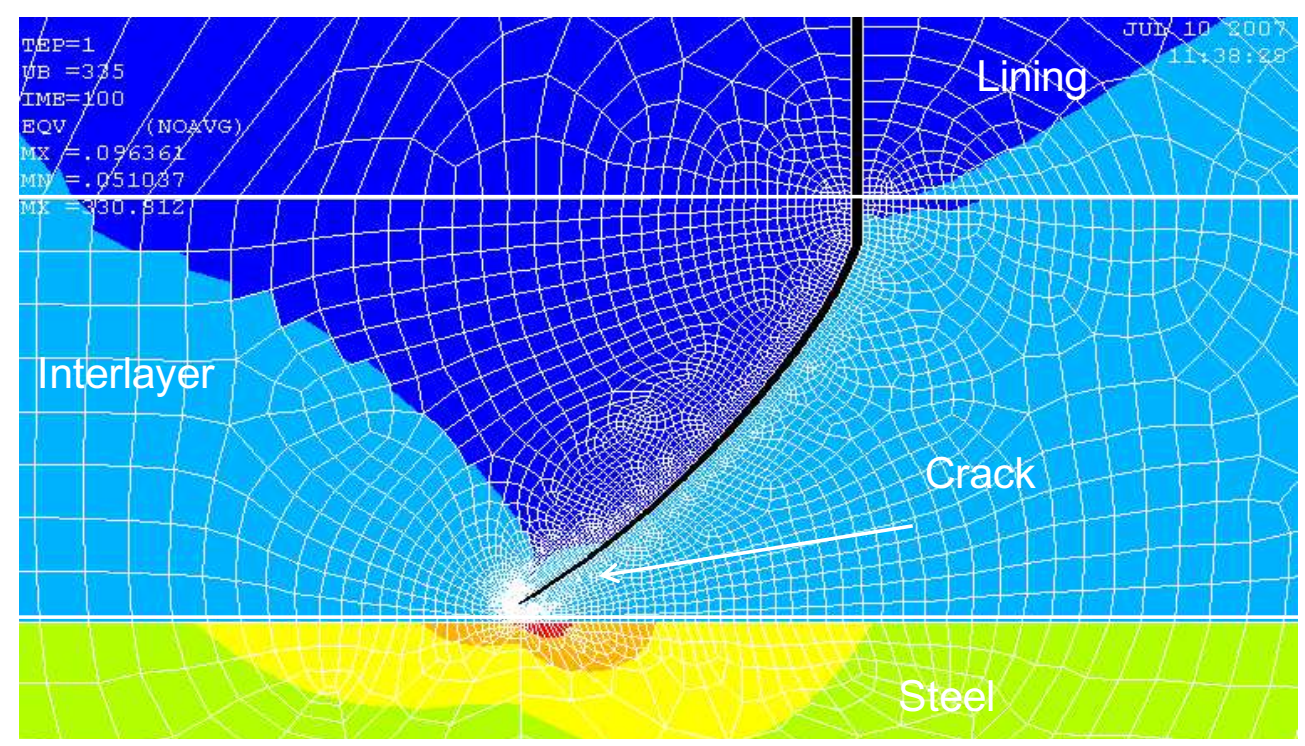


Figure 9

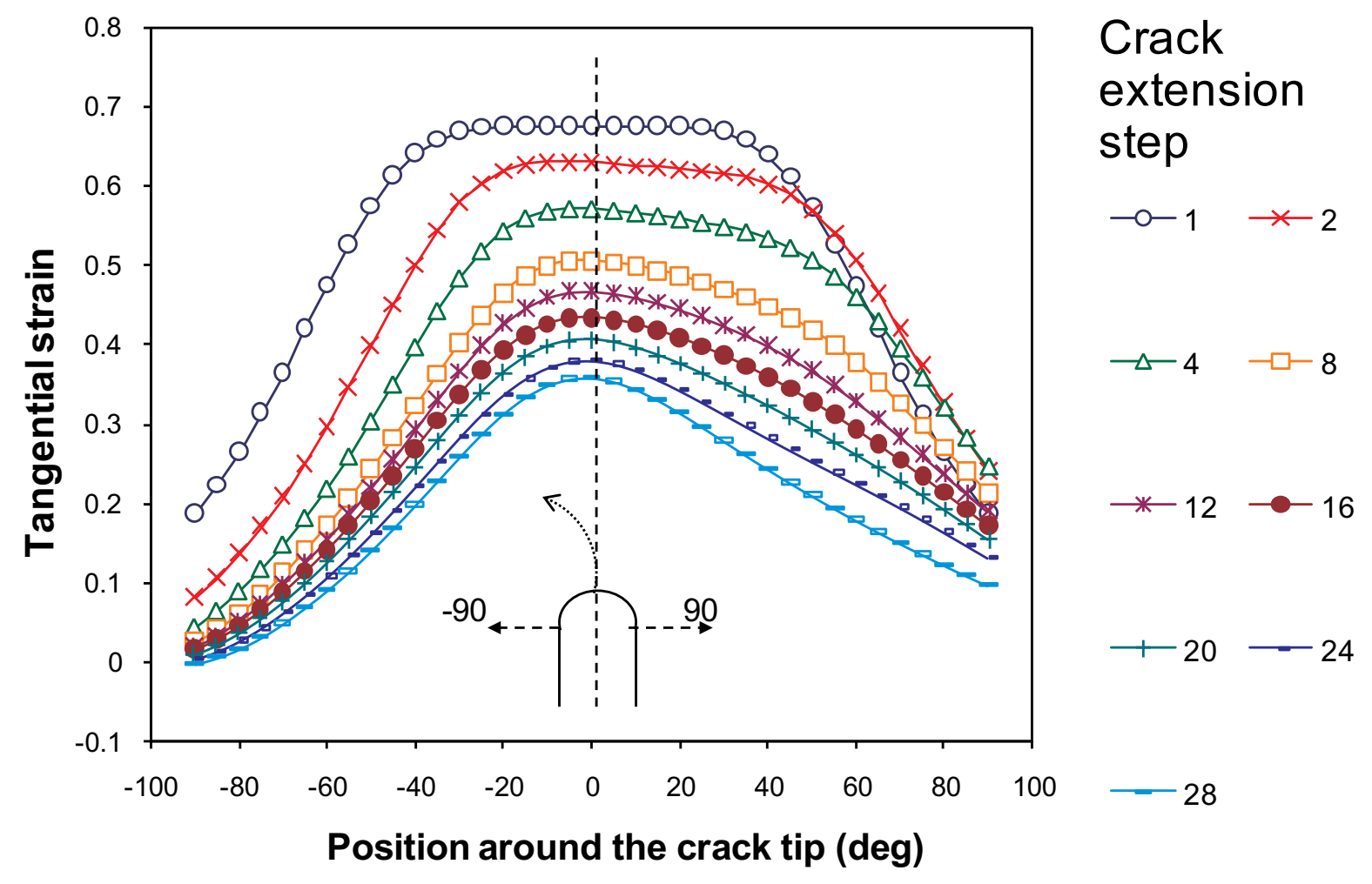


Figure 10

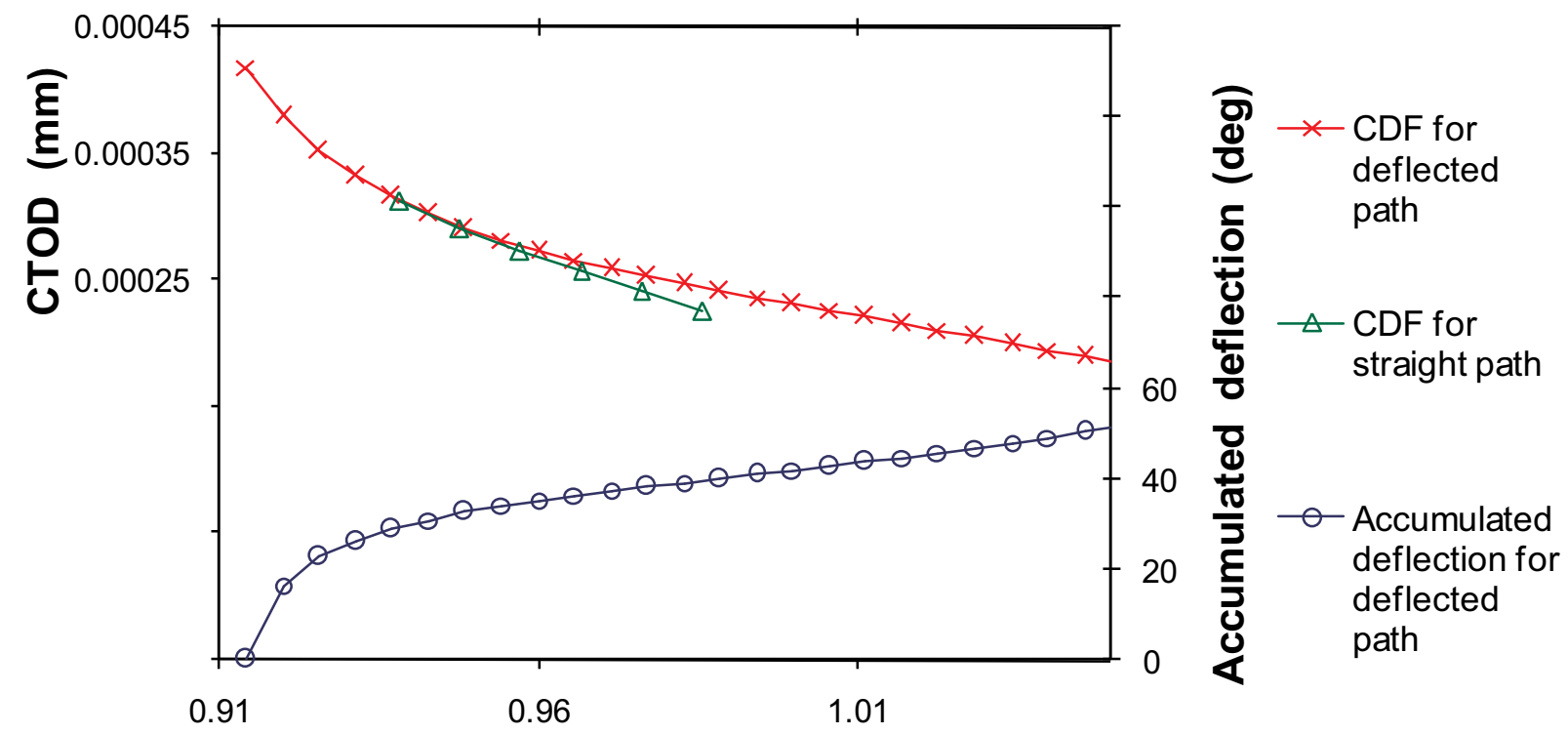

Normalised crack length $\left(a / t_{\mathrm{L} 2}\right)$ 


\section{Figure 11}

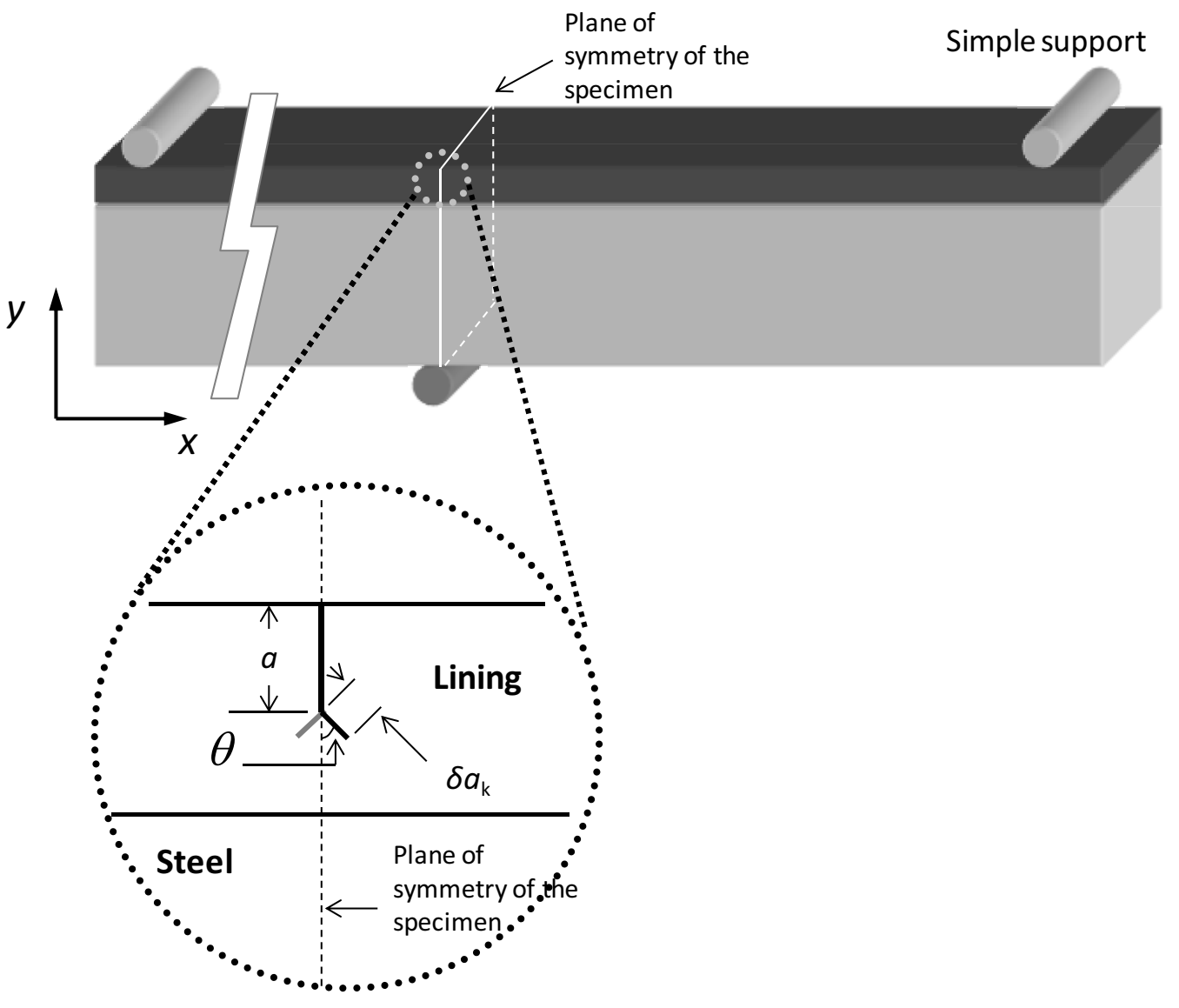


Figure 12

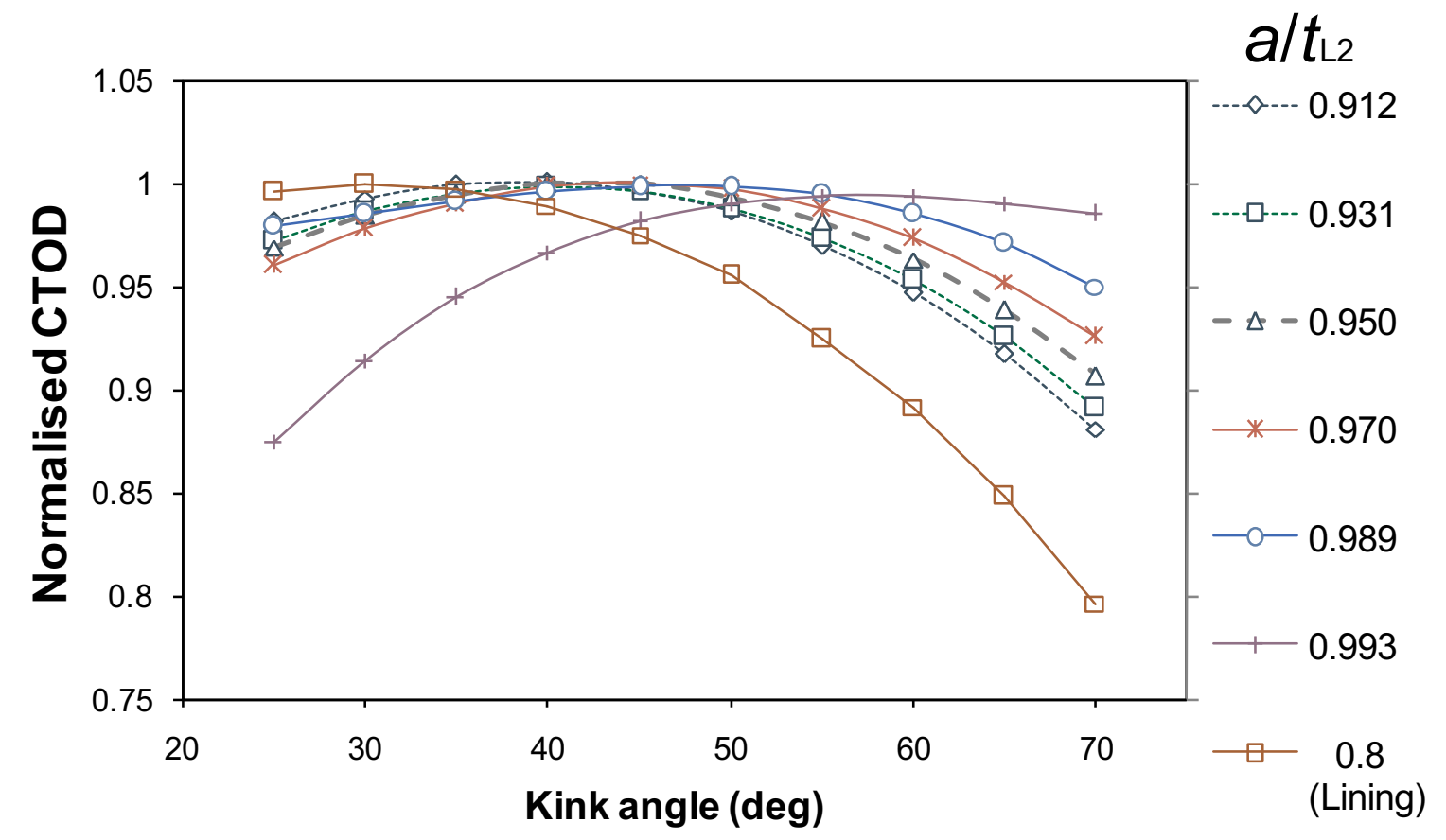


Figure 13

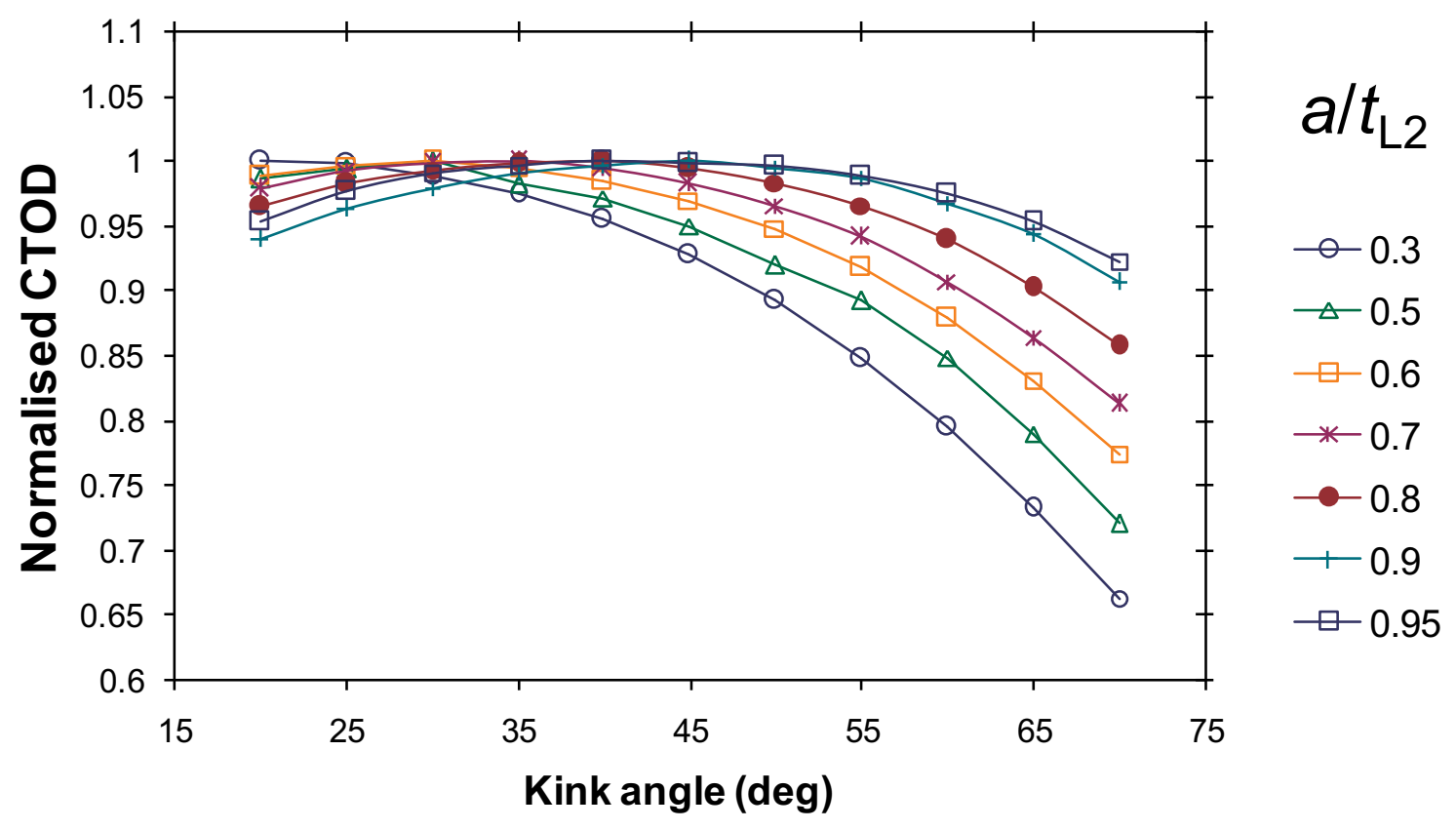

